\title{
Clinical features and prognostic analysis of patients with Aspergillus isolation during acute exacerbation of chronic obstructive pulmonary disease
}

\author{
Yu Gu ${ }^{1 \dagger}$, Xianping Ye ${ }^{1 \dagger}$, Yu Wang ${ }^{2}$, Kunlu Shen ${ }^{3}$, Jinjin Zhong ${ }^{2}$, Bilin Chen ${ }^{2}$ and Xin Su ${ }^{1,2,3^{*}}$
}

\begin{abstract}
Background: Lower respiratory tract (LRT) specimen culture is widely performed for the identification of Aspergillus. We investigated the clinical features and prognosis of patients with Aspergillus isolation from LRT specimens during acute exacerbation of chronic obstructive pulmonary disease (AECOPD).

Methods: This is a 6-year single-center, real-world study. 75 cases out of 1131 hospitalized AECOPD patients were positive for Aspergillus. These patients were carefully evaluated and finally diagnosed of pulmonary aspergillosis (PA, 60 cases, $80 \%$ ) or colonization (15 cases, 20\%). Comparisons of clinical data were performed between these two groups. A cox regression model was used to confirm prognostic factors of Aspergillus infection.
\end{abstract}

Results: The PA group had worse lung function and higher rates of systemic corticosteroid use and broad-spectrum antibiotic use before admission than the colonization group. The PA group had significantly higher in-hospital mortality and 180-day mortality than the colonization group (45\% (27/60) vs. $0 \%(0 / 15), p=0.001$, and $52.5 \%(31 / 59)$ vs. $6.7 \%$ $(1 / 15), p<0.001$, respectively). By multivariable analysis among Aspergillus infection patients, antifungal therapy (HR $0.383,95 \% \mathrm{Cl} 0.163-0.899, p=0.027)$ was associated with improved survival, whereas accumulated dose of systemic steroids $>700 \mathrm{mg}(\mathrm{HR} 2.452,95 \% \mathrm{Cl} 1.134-5.300, p=0.023$ ) and respiratory failure at admission (HR 5.983, 95\% Cl 2.487-14.397, $p<0.001$ ) were independently associated with increased mortality. Significant survival differential was observed among PA patients without antifungals and antifungals initiated before and after Aspergillus positive culture $(p=0.001)$.

Conclusions: Aspergillus isolation in hospitalized AECOPD patients largely indicated PA. AECOPD patients with PA had worse prognosis than those with Aspergillus colonization. Empirical antifungal therapy is warranted to improve the prognosis for Aspergillus infection.

Keywords: AECOPD, Aspergillus isolation, Pulmonary aspergillosis, Colonization, Prognosis

*Correspondence: suxinjs@163.com

${ }^{\dagger}$ Yu Gu and Xianping Ye contributed equally to this study

${ }^{1}$ Department of Respiratory and Critical Care Medicine, Jinling Hospital, Nanjing Medical University, 305 Zhongshan East Road, Xuanwu District, Nanjing 210002, China

Full list of author information is available at the end of the article

\section{Introduction}

Chronic obstructive pulmonary disease (COPD) is a common, preventable and treatable chronic respiratory disease characterized by airflow limitation and persistent respiratory symptoms. Airway microorganisms, including bacteria, viruses and fungi, play an important role in the pathogenesis of acute exacerbation of COPD (AECOPD) [1]. Recently, fungal infection and 
colonization, especially by Aspergillus spp., have been increasingly reported in COPD patients.

In some studies, the rates of isolation of Aspergillus spp. from COPD patients reached $16.6 \%$ and $14.1 \%$ during exacerbation and follow-up, respectively $[2,3]$. Isolation of Aspergillus from lower respiratory tract (LRT) samples may suggest temporary fungal passage, benign carriage, colonization, or infection $[4,5]$. Pulmonary aspergillosis (PA) is a life-threatening opportunistic infection. Depending on the immune status of patients and Aspergillus virulence, PA can be classified into different types, including allergic bronchopulmonary aspergillosis (ABPA), invasive pulmonary aspergillosis (IPA) and chronic pulmonary aspergillosis (CPA) [6]. Up to $22 \%$ of COPD patients with Aspergillus isolation from LRT samples have IPA [7]. IPA has a very poor prognosis in the COPD population, with a mortality rate as high as $71.7 \%$ [8].

Early recognition of PA is essential to decrease mortality and achieve a good outcome [9-11]. However, it is difficult to establish a PA diagnosis in patients with AECOPD. It is rarely feasible to perform lung biopsy in these patients [12]. LRT specimens are easier to collect than bronchoalveolar lavage fluid (BALF), which is widely used to detect Aspergillus. Distinguishing colonization from infection in AECOPD patients is still a challenge for clinicians.

The aim of this study was to describe clinical features of infection and colonization in AECOPD patients with positive Aspergillus isolation from LRT, and identify potential prognostic factors of Aspergillus infection on day-180 survival.

\section{Patients and methods}

\section{Patient recruitment}

This retrospective, real-world study was conducted among hospitalized patients with AECOPD who were admitted to Jinling Hospital (a tertiary teaching hospital with approximately $2500+$ beds) from January 2014 to December 2019. The study was approved by the ethics committee of Jinling Hospital. The informed consent was obtained from all participants in written form. A diagnosis of COPD was based on the Global Initiative for Chronic Obstructive Lung Disease guidelines [13]. Hospitalized AECOPD patients with at least one qualifying LRT specimen culture were screened and enrolled. Patients were excluded if a primary diagnosis of neutropenia $\left(<500\right.$ neutrophils $\left./ \mathrm{mm}^{3}\right)$ or hematologic malignancy was established. The medical records of these patients, including data on clinical manifestations, comorbidities, microbiology, radiology, treatment and survival within 180 days, were reviewed.

\section{Definitions of pulmonary aspergillosis and colonization}

The diagnosis of IPA was based on the Bulpa criteria [14]. Patients with proven and probable PA were enrolled. CPA was diagnosed according to the European Society for Clinical Microbiology and Infectious Diseases (ESCMID) and European Respiratory Society (ERS) guidelines [15]. Proven IPA was confirmed by the presence of hyphae compatible with Aspergillus in specimens taken from a pulmonary lesion through pulmonary biopsy within the previous 3 months. Probable IPA was diagnosed requiring three following criteria: (1) the use of steroids and routine treatment failure in severe AECOPD patients; (2) one of following microbiological evidence of Aspergillus, such as Aspergillus isolation from qualified LRT specimens, a positive serum Aspergillus antibody test or two sequential positive serum galactomannan (GM) tests; and (3) one or more of the following radiological imaging presentations: consolidation lesions or nodules with or without cavity formation, an air crescent sign or a halo sign. The definition of CPA was as follows: (1) persistent chronic respiratory or systemic symptoms, such as cough, hemoptysis, breathlessness, or weight loss, for at least 3 months; (2) the formation or progressive enlargement of one or more pulmonary cavities with or without a fungal ball or nodules on chest imaging; and (3) microbiological evidence of Aspergillus spp. from LRT specimens or the presence of positive Aspergillus-specific IgG; and (4) Aspergillus hyphae on histopathology or positive Aspergillus isolation from lung biopsy specimens. Bronchial lung cancer, pulmonary tuberculosis and other diseases with similar symptoms were excluded when histopathological evidence was lacking. Aspergillus colonization was defined as the positive isolation of Aspergillus from LRT specimens from patients who had no other supportive evidence of PA and recovered from AECOPD without antifungal therapy [14].

\section{Statistical analysis}

All statistical analyses were performed using SPSS statistics software (version 25). Qualitative variables are expressed as percent distributions in each category, and quantitative variables are expressed as means \pm standard deviations for normally distributed variables or medians (ranges) for nonnormally distributed variables. Pearson's chi-square test or Fisher's exact test was used to compare qualitative variables. Quantitative variables with normal distributions were compared with Student's t-test, while nonnormally distributed variables were compared with the Mann-Whitney U test.

Survival curves were used to analyze the prognosis of patients from admission to day 180. Survival analysis was performed with the Kaplan-Meier method with 
the log-rank test. A multivariable Cox analysis with forward selection was performed in Aspergillus infection to determine independent factors associated with day180 mortality. Variables with a $p$ value $<0.1$ in univariate analysis were included into the multivariate model. Finally, only variables with $p$ value $<0.05$ were retained in the multivariate model. Statistical tests were two-tailed, and a $\mathrm{P}$ value less than 0.05 was considered statistically significant.

\section{Results}

There were 1298 AECOPD patients admitted to our hospital from January 2014 to December 2019. One patient was excluded because of neutropenia, and 166 patients were excluded because LRT specimen culture was not performed. A total of 1131 patients had at least one qualified LRT specimen culture. Among these, Aspergillus was isolated from LRT specimens from 75 (6.6\%) patients. Sixty $(80 \%)$ out of 75 patients were finally diagnosed with proven IPA $(n=1)$, probable IPA $(n=55)$ and CPA $(n=4)$ and included in the PA group. One proven
IPA case was confirmed by computed tomography (CT)guided percutaneous lung biopsy. Fifteen (20\%) out of 75 patients were positive for colonization.

From the 75 PA patients, 400 cultures were performed, yielding 142 positive Aspergillus isolates from LRT specimens. Positive LRT specimens consisted of sputum $(\mathrm{n}=118)$ and bronchial aspirate $(\mathrm{n}=24)$. A. fumigatus was the most frequently recovered species $(\mathrm{n}=54,72 \%)$, followed by A. flavus $(\mathrm{n}=9,12 \%)$, A. niger $(\mathrm{n}=3,4 \%)$ and unclassified species (14, 18.7\%). Two species of Aspergillus were isolated from LRT specimens from five patients (A. fumigatus + A. flavus $(\mathrm{n}=3)$ and A. fumigatus $+A$. niger $(\mathrm{n}=2))$.

\section{Comparisons of clinical characteristics, laboratory tests and radiological presentations}

The clinical characteristics of patients with positive Aspergillus isolation are shown in Table 1. There was no significant difference between the two groups in terms of demographic data. The PA group had a higher proportion of patients with severe COPD (GOLD III-IV)

Table 1 Clinical characteristics of patients positive for Aspergillus isolation from LRT specimens

\begin{tabular}{|c|c|c|c|}
\hline Variables & $\begin{array}{l}\text { PA group } \\
n=60\end{array}$ & $\begin{array}{l}\text { Colonization group } \\
n=15\end{array}$ & P value \\
\hline Age (years, median) & $71.5(31,93)$ & $75(49,89)$ & 0.219 \\
\hline Sex (male), n (\%) & $54(90)$ & $14(93.3)$ & 1.000 \\
\hline GOLD III-IV, n (\%) & $53(88.3)$ & $9(60)$ & 0.018 \\
\hline \multicolumn{4}{|l|}{ Corticosteroids before admission, n (\%) } \\
\hline Inhaled & $45(75)$ & $9(60)$ & 0.335 \\
\hline Intravenous or oral & $31(51.7)$ & $3(20)$ & 0.028 \\
\hline Accumulated dose of systemic steroids $>700 \mathrm{mg}^{\mathrm{a}}$ & $11(18.3)$ & $1(6.7)$ & 0.439 \\
\hline Broad-spectrum antibiotics before admission ${ }^{\mathrm{b}}, \mathrm{n}(\%)$ & $45(75)$ & $3(20)$ & $<0.001$ \\
\hline Hospital stay (days, median) & $15.5(1-72)$ & $15(7-42)$ & 0.691 \\
\hline ICU admissionc, n (\%) & $31(51.7)$ & $3(20)$ & 0.028 \\
\hline Mechanical ventilation, n (\%) & $21(35)$ & $2(13.3)$ & 0.128 \\
\hline \multicolumn{4}{|l|}{ Clinical symptoms, n (\%) } \\
\hline Fever $^{d}$ & $39(65)$ & $4(26.7)$ & 0.007 \\
\hline Cough & $57(95)$ & $13(86.7)$ & 0.260 \\
\hline Sputum & $43(71.7)$ & $7(46.7)$ & 0.066 \\
\hline Hemoptysis & $4(6.7)$ & $2(13.3)$ & 0.593 \\
\hline Respiratory failure & $28(46.7)$ & $3(20)$ & 0.061 \\
\hline \multicolumn{4}{|l|}{ Comorbidities, n (\%) } \\
\hline Bronchiectasis & $10(16.7)$ & $2(13.3)$ & 1.000 \\
\hline Tuberculosis before & $11(18.3)$ & $3(20)$ & 1.000 \\
\hline Hypertension & $24(40)$ & $8(53.3)$ & 0.350 \\
\hline Diabetes mellitus & $20(33.3)$ & $2(13.3)$ & 0.205 \\
\hline Cardiac insufficiency & $20(33.3)$ & $3(20)$ & 0.369 \\
\hline Renal insufficiency ${ }^{\mathrm{e}}$ & $8(13.3)$ & $2(13.3)$ & 1.000 \\
\hline Solid malignant tumor & $5(8.3)$ & $3(20)$ & 0.193 \\
\hline
\end{tabular}

ICU intensive care unit. ${ }^{a}$ Dose of systemic steroids in prednisone equivalents before first Aspergillus isolation; ${ }^{b}$ three or more antibiotics in the past 3 months; ${ }^{\mathrm{C}} \mathrm{CCU}$ admission at any time during hospitalization; ${ }^{\mathrm{d}} \mathrm{T}>38^{\circ} \mathrm{C}$; ${ }^{\mathrm{e}}$ serum creatinine $>1.5 \mathrm{mg} / \mathrm{dL}$; significance of bold: a $\mathrm{P}$ value less than 0.05 was considered statistically significant 
than the colonization group $(p=0.018)$. The PA group also had a higher rate of admission to the intensive care unit (ICU) during hospitalization than the colonization group $(p=0.028)$. There was no significant difference in the length of hospital stay. More patients with Aspergillus infection required mechanical ventilation than patients with colonization ( $45 \%$ vs. $13.3 \%, p=0.024)$. PA patients had a higher rate of systemic corticosteroid use than colonization patients $(51.7 \%$ vs $20.0 \%, p=0.028)$. Prior to admission, the proportion of patients who received intravenous broad-spectrum antibiotics was higher in the PA group than in the colonization group $(75.0 \%$ vs $20.0 \%$, $p<0.001)$. The most common symptoms were cough $(\mathrm{n}=70,93.3 \%)$ and sputum production $(\mathrm{n}=50,66.7 \%)$, but no difference was observed between the two groups. Fever was more common in the PA group than in the colonization group ( $65 \%$ vs $26.7 \%, p=0.007$ ). Hemoptysis was uncommon in both groups. In terms of comorbidities, no significant difference was observed.

Laboratory and CT imaging data were included in Table 2. The laboratory data showed that inflammatory biomarkers including white blood cell (WBC) counts, neutrophil counts and C-reactive protein (CRP) levels significantly increased in the PA group. The level of procalcitonin (PCT) did not differ between the two groups. Patients with PA had significantly lower albu$\min$ (ALB) than patients with colonization ( $28.3 \pm 6.2$ vs. $32.3 \pm 6.5, p=0.029)$. Serum galactomannan (GM) detection was performed in 63 patients. Serum GM (cutoff $0.5)$ was found to be positive in $28(53.8 \%)$ cases in the PA group and 1 (6.7\%) patient in the colonization group. The patient with positive serum GM in the colonization group was finally diagnosed with colonization due to subsequent consecutive negative GM results, and the patient recovered from AECOPD without antifungal therapy. BALF GM detection was not routinely performed in this population. All BALF GM tests in 6 patients in the PA group were positive (cutoff 0.7 ). CT was performed in 67 (89.3\%) patients. The CT findings were not significantly different between the two groups. The results demonstrated that infiltration $(\mathrm{n}=62)$ was the most common radiological finding in patients with Aspergillus isolation from LRT specimens. Specific signs were rarely observed in AECOPD patients with PA; only 2 patients had halo signs and 3 patients had air crescent signs.

\section{Prognostic factors for PA patients and antifungal therapy} We followed 75 enrolled patients from admission to day 180. The clinical outcomes are presented in Table 3 . The total in-hospital and 180-day mortality rates were $36 \%$

Table 2 Laboratory and radiological data of patients with Aspergillus isolation from LRT specimens

\begin{tabular}{|c|c|c|c|}
\hline Variables & $\begin{array}{l}\text { PA group } \\
n=60\end{array}$ & $\begin{array}{l}\text { Colonization group } \\
n=15\end{array}$ & $P$ value \\
\hline \multicolumn{4}{|l|}{ Laboratory data $^{a}$} \\
\hline WBC count $\left(10^{9} / L\right)$ & $11.3(3.3-43.3)$ & $6.3(3.3-26.5)$ & 0.001 \\
\hline Neutrophil count $\left(10^{9} / \mathrm{L}\right)$ & $9.6(2.3-41.9)$ & $4.1(2.1-23.5)$ & 0.001 \\
\hline Lymphocyte count $\left(10^{9} / L\right)$ & $0.7(0.1-4.9)$ & $1.1(0.4-2.3)$ & 0.063 \\
\hline $\mathrm{Hb}(\mathrm{g} / \mathrm{L})$ & $117.7 \pm 19.3$ & $128.9 \pm 43.5$ & 0.345 \\
\hline $\operatorname{PLT}\left(10^{9} / \mathrm{L}\right)$ & $187(20,522)$ & $222(46,370)$ & 0.425 \\
\hline $\mathrm{ALB}(\mathrm{g} / \mathrm{L})$ & $28.3 \pm 6.2$ & $32.3 \pm 6.5$ & 0.029 \\
\hline $\mathrm{LDH}(\mathrm{U} / \mathrm{L})$ & $457.5(155-3126)$ & $269(168-1096)$ & 0.056 \\
\hline $\mathrm{CRP}(\mathrm{mg} / \mathrm{L})$ & $64.3(1.1-258.8)$ & $16.2(0-320)$ & 0.006 \\
\hline PCT (ug/L) & $0.2(0-16)$ & $0.1(0-9.6)$ & 0.195 \\
\hline Serum $\mathrm{GM}^{\mathrm{b}}, \mathrm{n}(\%)$ & 28/52 (53.8) & $1 / 11(9.1)$ & 0.007 \\
\hline BALF GMc,$n(\%)$ & $6 / 6(100)$ & / & \\
\hline Radiological data, n (\%) & $53(88.3)$ & 14 (93.3) & \\
\hline Consolidation & 20 & 3 & 0.349 \\
\hline Infiltration & 51 & 11 & 0.058 \\
\hline Nodules & 11 & 4 & 0.498 \\
\hline Cavitation & 8 & 0 & 0.189 \\
\hline Halo sign & 2 & 0 & 1.000 \\
\hline Air crescent sign & 3 & 0 & 1.000 \\
\hline Hydrothorax & 22 & 2 & 0.059 \\
\hline Pleural thickening & 30 & 4 & 0.062 \\
\hline
\end{tabular}

WBC white blood cell, $H b$ hemoglobin, $P L T$ platelet, $A L B$ albumin, $L D H$ lactate dehydrogenase, $C R P C$-reactive protein, $P C T$ procalcitonin, IL-6 interleukin-6, GM galactomannan, BALF bronchoalveolar lavage fluid; ${ }^{a} B$ Blood specimen; ${ }^{b}$ the cutoff value is $0.5 ;{ }^{c}$ the cutoff value is 0.7 ; significance of bold: a $P$ value less than 0.05 was considered statistically significant 
Table 3 In-hospital mortality and outcomes following 180 days in the PA group and colonization group

\begin{tabular}{|c|c|c|c|}
\hline Outcomes & $\begin{array}{l}\text { PA group } \\
n=60\end{array}$ & $\begin{array}{l}\text { Colonization } \\
\text { group } \\
n=15\end{array}$ & $P$ value \\
\hline In-hospital mortality, n (\%) & $27(45)$ & 0 & 0.001 \\
\hline Survival ${ }^{\mathrm{a}}, \mathrm{n}$ & 28 & 14 & \\
\hline Lost to follow-up ${ }^{b}$ & 1 & 0 & \\
\hline 180-day mortality, n (\%) & $31(52.5)$ & $1(6.7)$ & $<0.001$ \\
\hline Due to Aspergillosis & 21 & 0 & \\
\hline $\begin{array}{l}\text { Due to Aspergillosis and other } \\
\text { causes }\end{array}$ & 10 & 0 & \\
\hline Not due to Aspergillosis & 0 & 1 & \\
\hline
\end{tabular}

a Patients survived for 180 days until the end of follow-up. ${ }^{\text {b}}$ These patients could not be contacted because of incorrect or lost information. 'Death within 180 days after admission

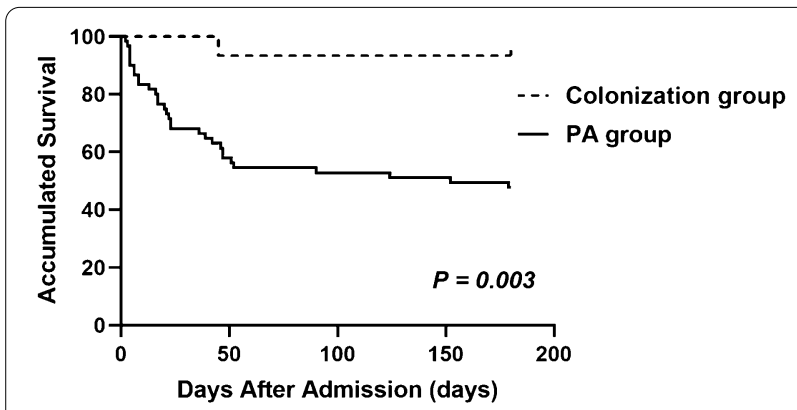

Fig. 1 Survival from admission to day 180 in patients in the PA and colonization groups according to Kaplan-Meier analysis with the log-rank test $(p=0.003)$
(27/75) and 43.2\% (32/74), respectively, in patients with Aspergillus isolation from LRT specimens. Both in-hospital and 180-day mortality were significantly higher in the PA group than in the colonization group $(45 \%$ vs. 0 and $52.5 \%$ vs. $6.7 \%, p=0.001, p<0.001$, respectively). Among 31 patients who died in the PA group, 21 died of aspergillosis, and 10 died of aspergillosis comorbid with other diseases (such as lung cancer, renal failure, or cardiac failure). One patient in the colonization group died of multiple organ dysfunction syndrome (MODS) after meningioma surgery. The Kaplan-Meier 180-day curve revealed that patients in the PA group (105 days; $95 \% \mathrm{CI}$ 85-125) had a shorter mean survival time than those in the colonization group (171 days; 95\% CI 154-188) (logrank, $p=0.003$ ) (Fig. 1). Notably, all patients who died in the PA group had IPA. By multivariable analysis, antifungal therapy (HR 0.383, 95\% CI $0.163-0.899, p=0.027$ ) was associated with improved survival, whereas accumulated dose of systemic steroids $>700 \mathrm{mg}$ (HR 2.452, 95\% CI 1.134-5.300, $p=0.023)$ and respiratory failure at admission (HR 5.983, 95\% CI 2.487-14.397, $p<0.001$ ) were independently associated with higher day-180 mortality (Fig. 2).

Antifungal treatment was administered in 85\% (51 out of 60 patients) of PA patients. PA patients were divided into 3 subgroups according to antifungal treatment time: 9 patients without antifungal therapy were considered subgroup 1, 10 patients who received antifungal therapy before Aspergillus isolation were considered subgroup 2 , and 41 patients who received antifungal therapy after positive Aspergillus isolation were considered subgroup 3 . The most commonly used antifungal drug was voriconazole $(46,76.7 \%)$, followed by caspofungin (24, $40 \%)$, amphotericin B $(4,6.7 \%)$, and itraconazole $(3,5 \%)$.

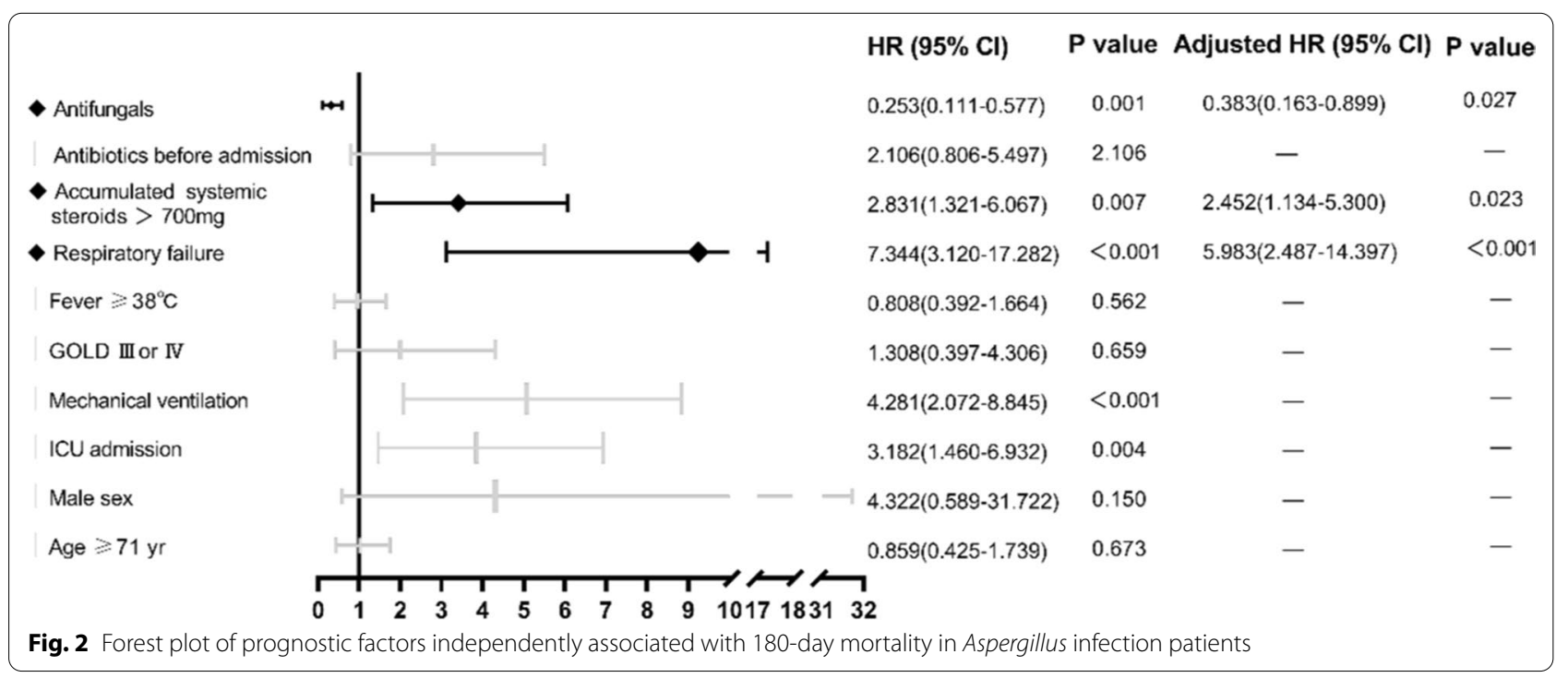




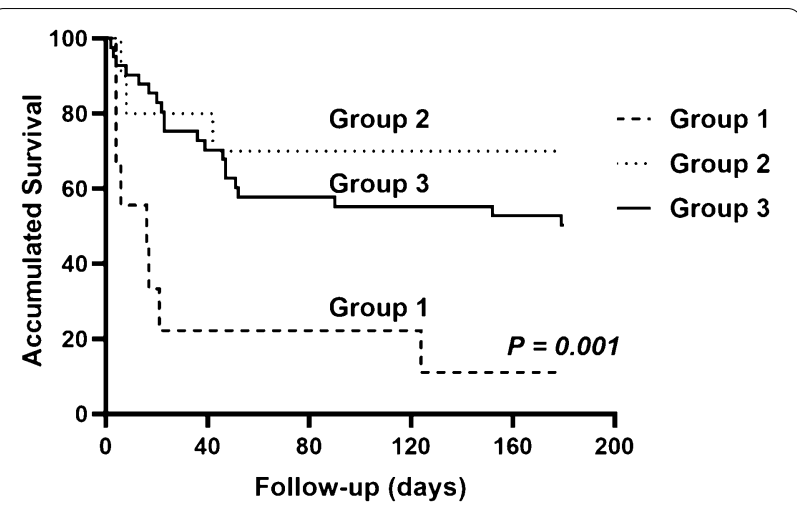

Fig. 3 Kaplan-Meier cumulative survival curve of PA patients in the 3 subgroups. Curves were compared with the log-rank test $(p=0.001)$

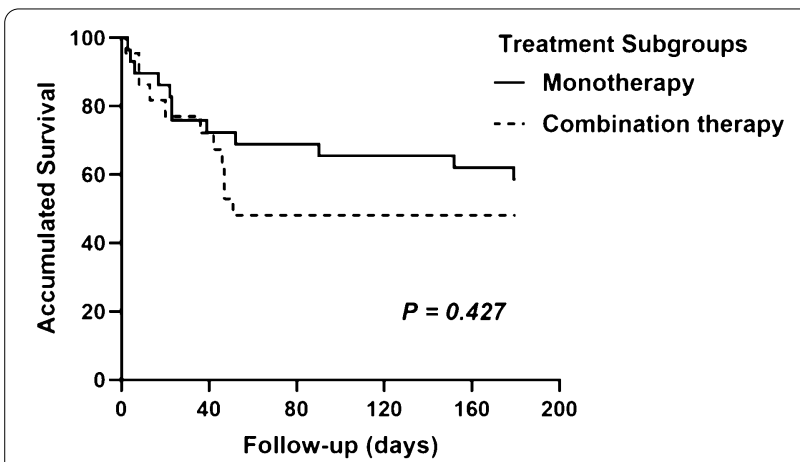

Fig. 4 Survival from admission to 180 day of patients with monotherapy and combination antifungal therapy by Kaplan-Meier analysis with log-rank test $(p=0.427)$

Among the three subgroups, the overall in-hospital mortality was $77.8 \%, 30 \%$ and $41.5 \%$, respectively $(p=0.094)$. There was a significant difference in 180-day mortality among the three subgroups $(88.9 \%$ vs $30 \%$ vs $50 \%$, $p=0.034$ ). During the follow-up period of 180 days, group 2 had a significantly higher cumulative survival rate than groups 1 and 3 (Fig. 3, $p=0.001$ ). Fifty-one PA patients treated with antifungals received monotherapy $(n=29)$ or combination therapy $(n=22)$. Twelve patients in the monotherapy group and 11 patients in the combination therapy group died during the follow-up period. Figure 4 showed no difference observed in the 180-day prognosis between the monotherapy and combination therapy groups (log-rank, $p=0.427)$.

\section{Discussion}

This real-world study investigated the clinical significance of Aspergillus spp. isolation from LRT specimens in a cohort of AECOPD patients considering three aspects: (1) the incidence of infection and colonization in patients with Aspergillus isolation from LRT specimens;
(2) differences between infection and colonization in terms of clinical characteristics; and (3) the prognostic factors of the Aspergillus infection and the effects of antifungals on prognosis.

It is important for physicians to distinguish infection from colonization in AECOPD patients when Aspergillus is isolated from LRT specimens since an early diagnosis is crucial to improve the prognosis [14]. Some studies have demonstrated that once admitted to the hospital, $1.3 \%-3.9 \%$ of patients with COPD develop culture-positive invasive aspergillosis, not always as a result of oral corticosteroid use [16]. Our study revealed that the LRT specimens from $6.63 \%$ of AECOPD patients were positive for Aspergillus, and most of them had Aspergillus infections, resulting in a PA incidence rate of $5.3 \%$ in hospitalized AECOPD patients. Among studies focused on COPD patients with Aspergillus isolated from LRT specimens, the actual prevalence of infection varied from 22.2 to $61.2 \%$ [7, 17, 18]. In our study, Aspergillus isolation from LRT specimens from AECOPD patients revealed that $80 \%$ of patients were infected with Aspergillus. Thus, Aspergillus isolation from LRT specimens in hospitalized AECOPD is important and meaningful. In most cases, isolation indicates Aspergillus infection rather than colonization.

Uncovering risk factors for infection is important in differentiating between infection and colonization [19]. In terms of clinical symptoms, only fever was significantly more common in the PA group than in the colonization group. Cough and sputum production were also observed in most AECOPD patients in this study. However, cough and sputum were not graded in this study. As a result, the actual differences may be underestimated.

Our study found that systemic corticosteroid use was more common in PA patients than in colonization patients. Many other studies have shown that the utility of corticosteroids plays a significant role in Aspergillus colonization [20] and the emergence of invasive aspergillosis [14] in patients with COPD, a high dose of corticosteroids is considered a risk factor for IPA [21, 22]. We reported that systemic steroids more than $700 \mathrm{mg}$ was not only possible risk factor for Aspergillus infection, but also associated with higher day-180 mortality.

This study illustrated that AECOPD patients with Aspergillus infection had a higher rate of broad-spectrum antibiotic use prior to admission than patients with colonization. This result was also found in other studies $[12,22]$. Antibiotic use may play a role in predisposing patients to Aspergillus colonization and infection. When antibiotic treatment fails in AECOPD patients, physicians should consider the probability of Aspergillus infection. Although the proportion of aspergillosis patients with GOLD III-IV lung function was higher than that 
of colonization patients, the relationship between lung function and Aspergillus infection remains unclear.

The efficiency of BALF and serum GM detection for the early diagnosis of Aspergillus infection has been clearly demonstrated in neutropenia patients [23]. A meta-analysis suggested that serum GM had a lower positivity rate in immunocompetent hosts (61-71\%) [24]. The data in our study seem to confirm that serum GM detection is not satisfactory. In nonneutropenic patients, BALF GM is more sensitive than serum GM $[25,26]$. Our previous study indicated that when the BALF GM cutoff value was set to $\geq 0.5$ or $\geq 1.0$, the positive-likelihood ratios of the BALF GM test were all higher than those of the serum GM test [27].

The timely initiation of antifungal treatment is important in invasive fungal disease (IFD) [23]. The beneficial effect was expected from antifungal therapy for Aspergillus infection in our cohort. Voriconazole is a first-line antifungal for pulmonary aspergillosis [28]. In our study, $76.7 \%$ of PA patients were treated with voriconazole. Although the prognosis of patients with monotherapy and combination therapy was not different in our study, combination antifungals are suggested for cases of severe invasive fungal infections [29].

It is well-known that IPA is a threatening disease, while few studies focus on significance of Aspergillus positive culture for diagnosing Aspergillus infection. Our study reminds clinicians of high incidence of AECOPD patients. Our results also put forward the need of antifungal treatment initiation for at-risk patients as soon as they receive steroids more than $700 \mathrm{mg}$ or present sign of respiratory failure at admission.

This real-world study has some limitations. First, not all COPD patients received underwent LRT specimen examinations, which limited the number of samples. Second, histopathological examination is necessary to confirm the diagnosis. However, AECOPD patients were not able to undergo invasive procedures due to poor lung function.

In summary, it is of great significance to isolate Aspergillus from LRT specimens from AECOPD patients. AECOPD patients with PA have a much higher risk of mortality than those with Aspergillus colonization. Antifungal treatment is associated with improved survival whereas systemic steroids and respiratory failure are associated with poor prognosis. Early diagnosis of PA by sensitive microbiological tests and an effective algorithm is pivotal to improve survival.

\section{Abbreviations}

LRT: Lower respiratory tract; AECOPD: Acute exacerbation of chronic obstructive pulmonary disease; PA: Pulmonary aspergillosis; HR: Hazard ratio; ABPA:

Allergic bronchopulmonary aspergillosis; IPA: Invasive pulmonary aspergillosis;
CPA: Chronic pulmonary aspergillosis; COPD: Chronic obstructive pulmonary disease; ECSMID: European Society for Clinical Microbiology and Infectious Diseases; ERS: European Respiratory Society; GM: Galactomannan; CT: Computed tomography; GOLD: Global initiative for chronic obstructive lung disease; ICU: Intensive care unit; WBC: White blood cell; CRP: C-reactive protein; PCT: Procalcitonin; ALB: Albumin; BALF: Bronchoalveolar lavage fluid; Cl: Confidence interval.

\section{Acknowledgements}

Not applicable.

\section{Authors' contributions}

Xin Su, Yu Gu and Xianping Ye took responsibility for designing the study, acquiring most data, analyzing results and drafting the main manuscript text. Yu Wang and Kunlu Shen prepared Figs. 1-4. Jinjin Zhong and Bilin Chen contributed to collect part of primary data. Xin Su provided guidance for implementation and completion of the study. All authors reviewed and approved the manuscript.

\section{Funding}

This work was supported by the Project of Natural Science Foundation of China [Grant Number 82070011, 81873400 to Dr. Su], the Key Project of Jiangsu Commission of Health (K2019004 to Dr. Su), and the "333 project" of Jiangsu Province (BRA2019339 to Dr. Su). The funding body had no role in the design of the study and collection, analysis, and interpretation of data and in writing the manuscript.

\section{Availability of data and materials}

The datasets used and/or analysed during the current study are available from the corresponding author on reasonable request.

\section{Ethics approval and consent to participate}

The study was approved by the ethics committee of Jinling Hospital. The informed consent was obtained from all participants in written form. All methods were performed in accordance with relevant guidelines and regulations.

\section{Consent for publication}

Not applicable.

\section{Competing interests}

The authors have no competing interest to declare.

\section{Author details}

${ }^{1}$ Department of Respiratory and Critical Care Medicine, Jinling Hospital, Nanjing Medical University, 305 Zhongshan East Road, Xuanwu District, Nanjing 210002, China. ${ }^{2}$ Department of Respiratory and Critical Care Medicine, Jinling Hospital, Medical School of Nanjing University, Nanjing 210002, China.

${ }^{3}$ Department of Respiratory and Critical Care Medicine, Jinling Hospital, The First School of Clinical Medicine, Southern Medical University, Guangzhou 210002, China.

Received: 3 December 2020 Accepted: 31 January 2021

Published online: 26 February 2021

\section{References:}

1. Leung JM, Tiew PY, Mac Aogain M, Budden KF, Yong VF, Thomas SS, Pethe $\mathrm{K}$, Hansbro PM, Chotirmall SH. The role of acute and chronic respiratory colonization and infections in the pathogenesis of COPD. Respirology. 2017;22(4):634-50.

2. Huerta A, Soler N, Esperatti M, Guerrero M, Menendez R, Gimeno A, Zalacain R, Mir N, Aguado JM, Torres A. Importance of Aspergillus spp. isolation in Acute exacerbations of severe COPD: prevalence, factors and follow-up: the FUNGI-COPD study. Respir Res. 2014;15:17.

3. Chotirmall SH, Al-Alawi M, Mirkovic B, Lavelle G, Logan PM, Greene CM, McElvaney NG. Aspergillus-associated airway disease, inflammation, and the innate immune response. Biomed Res Int. 2013;2013:723129.

4. Bouza E, Guinea J, Pelaez T, Perez-Molina J, Alcala L, Munoz P. Workload due to Aspergillus fumigatus and significance of the organism 
in the microbiology laboratory of a general hospital. J Clin Microbiol. 2005;43(5):2075-9.

5. Horvath JA, Dummer S. The use of respiratory-tract cultures in the diagnosis of invasive pulmonary aspergillosis. Am J Med. 1996;100(2):171-8.

6. Soubani $\mathrm{AO}, \mathrm{Chandrasekar} \mathrm{PH}$. The clinical spectrum of pulmonary aspergillosis. Chest. 2002;121(6):1988-99.

7. Guinea J, Torres-Narbona M, Gijon P, Munoz P, Pozo F, Pelaez T, de Miguel J, Bouza E. Pulmonary aspergillosis in patients with chronic obstructive pulmonary disease: incidence, risk factors, and outcome. Clin Microbiol Infect. 2010;16(7):870-7.

8. Dai Z, Zhao H, Cai S, Lv Y, Tong W. Invasive pulmonary aspergillosis in nonneutropenic patients with and without underlying disease: a single-centre retrospective analysis of 52 subjects. Respirology. 2013;18(2):323-31.

9. Caillot D, Casasnovas O, Bernard A, Couaillier JF, Durand C, Cuisenier B, Solary E, Piard F, Petrella T, Bonnin A, et al. Improved management of invasive pulmonary aspergillosis in neutropenic patients using early thoracic computed tomographic scan and surgery. J Clin Oncol. 1997;15(1):139-47.

10. Salzer HJ, Cornely OA. Awareness of predictors of mortality may help improve outcome in chronic pulmonary aspergillosis. Eur Respir J. 2017;49(2):1602520.

11. Nivoix Y, Velten M, Letscher-Bru V, Moghaddam A, Natarajan-Ame S, Fohrer C, Lioure B, Bilger K, Lutun P, Marcellin L, et al. Factors associated with overall and attributable mortality in invasive aspergillosis. Clin Infect Dis. 2008;47(9):1176-84

12. He H, Ding L, Li F, Zhan Q. Clinical features of invasive bronchial-pulmonary aspergillosis in critically ill patients with chronic obstructive respiratory diseases: a prospective study. Crit Care. 2011;15(1):R5.

13. Vestbo J, Hurd SS, Agusti AG, Jones PW, Vogelmeier C, Anzueto A, Barnes PJ, Fabbri LM, Martinez FJ, Nishimura M, et al. Global strategy for the diagnosis, management, and prevention of chronic obstructive pulmonary disease: GOLD executive summary. Am J Respir Crit Care Med. 2013;187(4):347-65.

14. Bulpa P, Duplaquet F, Dimopoulos G, Vogelaers D, Blot S. Invasive Pulmonary Aspergillosis in Chronic Obstructive Pulmonary Disease Exacerbations. Semin Respir Crit Care Med 2020.

15. Denning DW, Cadranel J, Beigelman-Aubry C, Ader F, Chakrabarti A, Blot S, Ullmann AJ, Dimopoulos G, Lange C, et al. Chronic pulmonary aspergillosis: rationale and clinical guidelines for diagnosis and management. Eur Respir J. 2016;47(1):45-68

16. Denning DW. The ambitious " $95-95$ by 2025 " roadmap for the diagnosis and management of fungal diseases. Thorax. 2015;70(7):613-4.

17. Xu H, Li L, Huang WJ, Wang LX, Li WF, Yuan WF. Invasive pulmonary aspergillosis in patients with chronic obstructive pulmonary disease: a case control study from China. Clin Microbiol Infect. 2012;18(4):403-8.

18. He HY, Chang S, Ding L, Sun B, Li F, Zhan QY. Significance of Aspergillus spp. isolation from lower respiratory tract samples for the diagnosis and prognosis of invasive pulmonary aspergillosis in chronic obstructive pulmonary disease. Chin Med J Engl. 2012;125(17):2973-8.

19. Benedict K, Richardson M, Vallabhaneni S, Jackson BR, Chiller T. Emerging issues, challenges, and changing epidemiology of fungal disease outbreaks. Lancet Infect Dis. 2017;17(12):e403-11.

20. Tong $X$, Cheng A, Xu H, Jin J, Yang Y, Zhu S, Li Y. Aspergillus fumigatus during COPD exacerbation: a pair-matched retrospective study. BMC Pulm Med. 2018;18(1):55.

21. Leav BA, Fanburg B, Hadley S. Invasive pulmonary aspergillosis associated with high-dose inhaled fluticasone. N Engl J Med. 2000;343(8):586.

22. Muquim A, Dial S, Menzies D. Invasive aspergillosis in patients with chronic obstructive pulmonary diseases. Can Respir J. 2005:12(4):199-204.

23. Mengoli C, Cruciani M, Barnes RA, Loeffler J, Donnelly JP. Use of PCR for diagnosis of invasive aspergillosis: systematic review and meta-analysis. Lancet Infect Dis. 2009;9(2):89-96.

24. Pfeiffer CD, Fine JP, Safdar N. Diagnosis of invasive aspergillosis using a galactomannan assay: a meta-analysis. Clin Infect Dis. 2006:42(10):1417-27.

25. Patterson TF, Thompson GR 3rd, Denning DW, Fishman JA, Hadley S, Herbrecht R, Kontoyiannis DP, Marr KA, Morrison VA, Nguyen MH, et al. Practice guidelines for the diagnosis and management of Aspergillosis: 2016 Update by the Infectious Diseases Society of America. Clin Infect Dis. 2016;63(4):e1-60.

26. Jarvis JN, Percival A, Bauman S, Pelfrey J, Meintjes G, Williams GN, Longley $\mathrm{N}$, Harrison TS, Kozel TR. Evaluation of a novel point-of-care cryptococcal antigen test on serum, plasma, and urine from patients with HIV-associated cryptococcal meningitis. Clin Infect Dis. 2011;53(10):1019-23.

27. Zhou W, Li H, Zhang Y, Huang M, He Q, Li P, Zhang F, Shi Y, Su X. Diagnostic value of galactomannan antigen test in serum and bronchoalveolar lavage fluid samples from patients with nonneutropenic invasive pulmonary aspergillosis. J Clin Microbiol. 2017;55(7):2153-61.

28. Denning DW, Ribaud P, Milpied N, Caillot D, Herbrecht R, Thiel E, Haas A, Ruhnke M, Lode H. Efficacy and safety of voriconazole in the treatment of acute invasive aspergillosis. Clin Infect Dis. 2002;34(5):563-71.

29. Nivoix Y, Zamfir A, Lutun P, Kara F, Remy V, Lioure B, Rigolot JC, Entz-Werle $\mathrm{N}$, Letscher-Bru V, Waller J, et al. Combination of caspofungin and an azole or an amphotericin B formulation in invasive fungal infections. J Infect. 2006;52(1):67-74

\section{Publisher's Note}

Springer Nature remains neutral with regard to jurisdictional claims in published maps and institutional affiliations.
Ready to submit your research? Choose BMC and benefit from:

- fast, convenient online submission

- thorough peer review by experienced researchers in your field

- rapid publication on acceptance

- support for research data, including large and complex data types

- gold Open Access which fosters wider collaboration and increased citations

- maximum visibility for your research: over 100M website views per year

At BMC, research is always in progress.

Learn more biomedcentral.com/submissions 